\title{
Development and Evaluation of the Panoramic Night Vision Goggle
}

\author{
J. Craig, L. Task \\ Armstrong Laboratory, Wright-Patterson AFB OH \\ D. Filipovich \\ Night Vision Corporation, Lincolnwood IL
}

\begin{abstract}
$\underline{\text { Abstract }}$
A novel approach to significantly increasing the field of view (FOV) of night vision goggles (NVGs) has been developed and demonstrated. This approach uses four image intensifier tubes instead of the usual two to produce a very wide 100 degree horizontal by about 40 degree vertical FOV. A conceptual working model, designated the Panoramic NVG, has been fabricated and evaluated.
\end{abstract}

\section{Introduction}

The Panoramic NVG (PNVG) [1], [2] is a revolutionary change to traditional image intensifier-based night vision devices. The initial focus of the PNVG project centered around developing an "enhanced capability" NVG. A primary candidate parameter for enhancement was the NVG FOV with other parameters such as resolution, weight, center of gravity, and integrated display symbology overlay as secondary objective enhancements.

A conceptual working model was developed and fabricated (Figure 1) that displays a 100 degree horizontal by 40 degree vertical intensified FOV (Figure 2). This increased the intensified image seen by the wearer by 160 percent compared to the currently fielded 40 degree circular field of view systems. The larger FOV was achieved by using four off-the-shelf image intensifier tubes to produce four ocular channels. Two channels were used to produce a full 30 degree by 40 degree binocular FOV, and the other two were used to produce monocular left and right eye channels of about 35 degrees by
40 degrees. The PNVG's folded optical system resulted in a much better center of gravity compared to the currently fielded AN/AVS-6 and AN/AVS-9 type NVG configuration. Even with the added image intensifier tubes and associated optics, the overall weight of the device was comparable to currently fielded NVGs. The larger FOV and better center of gravity should reduce fatigue effects during long missions and potentially permit the PNVG to be retained upon ejection for use in evasion, escape, and rescue.

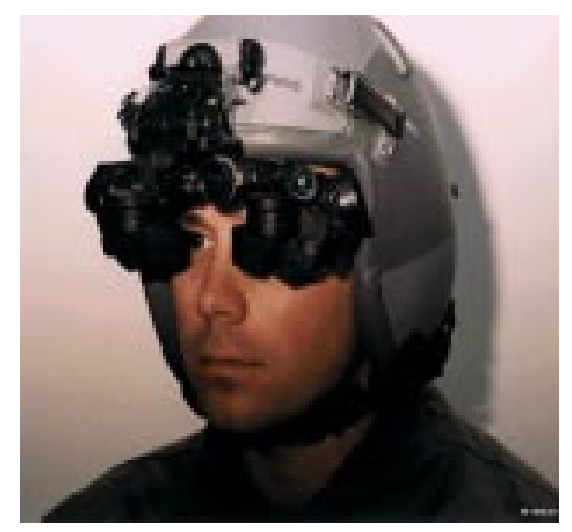

Fig. 1 PNVG Conceptual Working Model

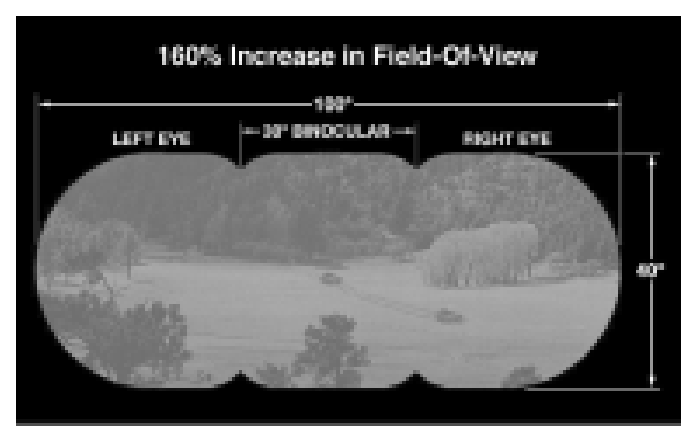

Fig. 2 PNVG simulated FOV 


\section{Background}

NVGs have been used in military aviation for more than 20 years with FOVs ranging from 30 degrees (early Cat's Eyes NVGs from GEC-Marconi Avionics) to 45 degrees (NITE-OP and NITE-Bird NVGs, also GEC-Marconi Avionics). The vast majority of NVGs used in military aviation have a 40 degree field of view (Figure 3) (AN/AVS-6 and AN/AVS-9). One major design characteristic of these NVGs is that increased FOV could only be obtained at the expense of resolution [3], [4] since each ocular uses only a single image intensifier tube. The image intensifier tube has a fixed number of pixels (picture elements). Therefore, if these are spread over a larger FOV, then the angular subtense per pixel increases, which corresponds to reduced resolution.

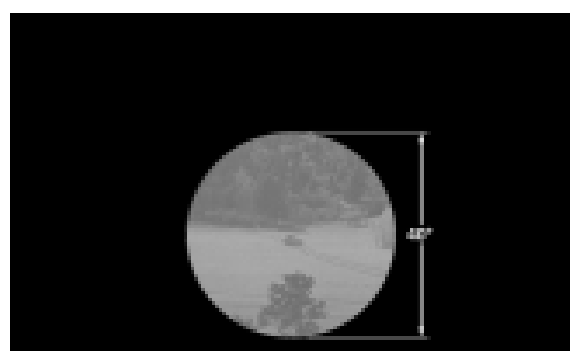

Fig. 3 Simulated AN/AVS-6 and AN/AVS-9 FOV for comparison with the PNVG

A fairly extensive survey of military (US Air Force) NVG users during 1992 and 1993 determined that increased FOV was the number one enhancement most desired by aircrew members closely followed by resolution [5], [6]. This was one of the major motivating factors in seeking an enhanced NVG capability.

\section{PNVG Description}

The PNVG features a partial overlap, 100 degree horizontal by approximately 40 degree vertical intensified FOV. The central 30 degree horizontal by 40 degree vertical FOV is completely binocular, while the right 35 degrees is seen with the right eye only and the left 35 degrees is viewed by the left eye only. A thin demarcation line separates the binocular scene from the outside monocular scenes. For this effort, four off-the-shelf PVS-7, third-generation, $18 \mathrm{~mm}$ format, image intensifier tubes were used. These tubes were selected because they do not have an image-inverting fiber optics attached. Using dual fixed eyepieces, tilted and fused together, folded optics, and a total of four adjustable-focus objective lenses, it was possible to produce the PNVG conceptual working model. Only $16 \mathrm{~mm}$ of the active $18 \mathrm{~mm}$ format of the image intensifier tube was used, which enabled the corresponding optics to be significantly reduced. This smaller format and the use of remote AN/AVS-6 power supplies were the primary reasons the PNVG weight was kept to only 569 grams, very similar to the currently fielded AN/AVS-6 and AN/AVS-9 NVG systems. Due to space constraints, slower than desired F/1.44 inner objective lenses and F/1.7 outer objective lenses were incorporated along with an eyepiece effective focal length of $21.9 \mathrm{~mm}$. Physical eye relief was measured to be approximately $17 \mathrm{~mm}$ (Figure 4).

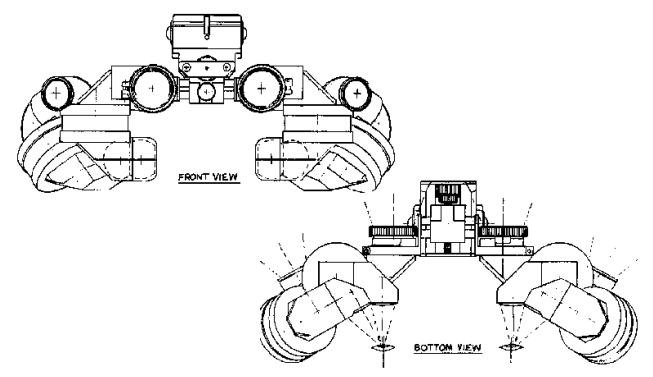

Fig. 4 Schematic diagram of the PNVG

A single inter-pupilary distance adjustment enables the wearer to align the eyepieces for their individual requirements. System power (AA alkaline batteries or standard military batteries) and mechanical mounting hardware are identical to the fielded systems thereby allowing for simple operation and compatibility for attachment, stow, and detachment of the PNVG. 


\section{$\underline{\text { PNVG Evaluation }}$}

Laboratory assessments were done on the PNVG for visual acuity under various illumination conditions (for the binocular FOV only) and for total visible FOV. To assess visual acuity, three trained observers looked through the PNVG at a chart composed of patches of square-wave gratings in a series of increasing spatial frequencies. The observer selected the highest spatial frequency grating that he/she could resolve then walked backward until the selected grating was barely resolvable. The baseline observation distance for the chart was 30 feet. The final angular spatial frequency of the grating (cycles/degree) was calculated by multiplying the base spatial frequency times the ratio of the walk-back distance to 30 feet. These data were then converted to cycles per milliradian (in parentheses below) and to Snellen acuities (numbers in front of the numbers in parentheses). This was done three times for each observer for each illumination condition. Each ocular of the PNVG was measured separately then a binocular assessment was made. The results shown below correspond to the median visual acuity (or resolution in cycles per milliradian) value obtained from the three observers:

MedianVisual Acuity (Resolution)

"Starlight Conditions"

$\frac{\text { Left channel }}{93(0.37)} \frac{\text { Rt. channel }}{93(0.37)} \frac{\text { Binocular }}{93(0.37)}$
"Quarter Moon Conditions"
$\frac{\text { Left channel }}{44(0.78)} \quad \frac{\text { Rt. channel }}{40(0.86)} \quad \frac{\text { Binocular }}{42(0.82)}$

Testing FOV was a bit more difficult due to the very large FOV and the nature of the partial binocular overlap. The PNVGs were positioned in a mount fixed to an optical bench, and observers viewed through the PNVGs to determine how far they could see to the edge of the FOV for each ocular. This spot was marked on the wall and trigonometry was used to determine the field angles. Since the far wall used for this assessment was only about 12 feet away, precise results could not be obtained. The median FOV values for the three observers are shown below:

Total FOV Per Channel

\begin{tabular}{lc} 
Channel & Median FOV degrees \\
\cline { 2 - 2 } Left outer & 42 \\
Left center & 33 \\
Rt. center & 35 \\
Rt. outer & 41 \\
Total Span & 92
\end{tabular}

** Note that after testing, bent brackets on the PNVG were discovered, which accounted for lower than expected readings for FOV. Follow-on measurements by the Night Vision Corporation indicated a total span of about 101 degrees.

Field evaluations have consisted primarily of "quick look" flight tests and ground assessments. The flight tests were accomplished on both Air Force and Army fixed- and rotary-wing platforms but limited to dry, high illumination conditions. Ground assessments were mainly done in darkrooms simulating the night environment. Final reports have not yet been published documenting the official findings. However, comments regarding the PNVG and the greatly enhanced field of view have been mostly positive. Some researchers were concerned that "luning", or other effects associated with the "eyedivergent" partial overlap method of obtaining the large total FOV, might be a problem [7]. However, no comments were received indicating this phenomena was observed.

\section{Future Directions}

Ten new working models in three different configurations are currently under development as part of an advanced technology demonstration. Configuration 1 (four each) will be designed for ejection compatibility. The PNVG will be attached to the HGU-55/P helmet, fit underneath the visor, and mounted to a "universal 
connector" used in the Visually-Coupled Acquisition and Targeting System helmetmounted display. Additionally, it will be equipped with an integrated head-up display (HUD) along with head tracker sensors and associated cables/electronic components. Configuration 2 (two each) will be designed for transport and rotary platforms. This configuration will attach to the HGU-55/P and SPH-4 helmet, mounted to the currently fielded AN/AVS-6 and AN/AVS-9 attachments, and will be equipped with the integrated HUD. Configuration 3 (four each) will be the same as configuration 2 but without the integrated HUD.

The primary objectives in this phase are to develop a $16 \mathrm{~mm}$ format image intensifier tube and associated power supply; integrate an active matrix electroluminescent display for symbology purposes; optimize the folded optical design as well as the human engineering of the overall packaging, mechanical adjustments, and attachments. Additionally, four weight and space models will be fabricated and subjected to windblast, impact, penetration, ballistic, and ejection tower (if required) testing.

\section{$\underline{\text { Conclusion }}$}

The PNVG conceptual working model demonstrates the feasibility and benefits of a very wide FOV image for night operations. Better situational awareness, reduced fatigue during long missions, possible ejection compatibility, and an overall increase in mission safety and effectiveness indicate the PNVG should have a tremendous impact on nighttime performance.

\section{Acknowledgments}

This work was funded by the Small Business Innovative Research program and the Armstrong Laboratory's Helmet-Mounted Sensory Technologies Program Office

\section{$\underline{\text { References }}$}

[1] Filipovich, D., Panoramic Night Vision System; Patent Pending.

[2] Filipovich, D., Visor-Mounted Night Vision System; Patent \# 5,416,315 (16 May 95)

[3] Donohue-Perry, M. M., Task, H. L., \& Dixon, S. A. (1994) Visual Acuity vs. Field of View and Light Level for Night Vision Goggles. Proceedings of SPIE Conference No.2218 Helmet- and HeadMounted Displays and Symbology Design Requirements, Orlando, FL, April, 1994.

[4] Task, H. L. (1992). Night vision devices and characteristics. AGARD Lecture Series 187: Visual Problems in Night Operations (pp. 7-1 - 7-8). Neuilly Sur Seine, France: NATO Advisory Group for Aerospace Research \& Development. (NTIS No. AGARD-LS-187)

[5] Hettinger, L. J., Donohue-Perry, M. M., Riegler, J. T., \& Davis, S. A. (1993). Night vision goggle (NVG) users' concerns survey site report: Fairchild AFB WA (Report No. AL/CF-TR-1993-0094). Wright-Patterson AFB, OH: Armstrong Laboratory. (DTIC No. B178368)

[6] Donohue-Perry, M. M., Hettinger, L. J., Riegler, J. T., \& Davis, S. A. (1993). Night vision goggle (NVG) users' concerns survey site report: Dover AFB DE (Report No. AL/CF-TR-1993-0075). WrightPatterson AFB, OH: Armstrong Laboratory. (DTIC No. B178369)

[7] Melzer, J. E. \& Moffitt, K. W. (1991). Ecological approach to partial binocular overlap. Large Screen Projection, Avionic, and Helmet-Mounted Displays, Proceedings of the SPIE, 1456, 124. 